\title{
In vivo fluoroscopic kinematography of cranio-caudal stifle stability after tibial tuberosity advancement (TTA): a retrospective case series of 10 stifles
}

\author{
Maartje Schwede ${ }^{1}$, Janna Rey ${ }^{1}$ and Peter Böttcher ${ }^{2, *}$ \\ ${ }^{I}$ Department of Small Animal Medicine, Faculty of Veterinary Medicine, University of Leipzig, An den Tierkliniken \\ 23, 04103 Leipzig, Germany \\ ${ }^{2}$ Small Animal Clinic, Free University of Berlin, Oertzenweg 19b, 14163 Berlin, Germany
}

\begin{abstract}
It was the aim of the study to determine retrospectively cranio-caudal stifle instability following TTA (tibial tuberosity advancement) using fluoroscopic kinematography. Ten stifles (eight dogs, mean body weight $27.3 \mathrm{~kg}$ ) with complete rupture of the cranial cruciate ligament and a mean follow-up of 12.8 weeks (5.4 - 28.4 weeks) after TTA underwent latero-lateral, uniplanar fluoroscopic kinematography while walking on a treadmill. Immediately before TTA, each stifle was explored arthroscopically and in the case of a longitudinal or bucket-handle tear of the caudal horn of the medial meniscus the unstable axial portion was resected. The high-speed fluoroscopic video sequences obtained were inspected visually for femoro-tibial translation (cranial drawer). The influence of postoperative patellar tendong angle (PTA), cage size and meniscal surgery on stifle stability was analyzed using logistic regression analysis. In three stifles, resection of unstable meniscal tissue was necessary. Fluoroscopically, nine out of ten stifles showed craniocaudal instability. Three stifles were potentially overcorrected (post PTA $<90^{\circ}$ ), seven potentially undercorrected (post PTA $>90^{\circ}$ ). None of the three parameters analyzed had a significant influence on postoperative in vivo stability $(\mathrm{p}=0.0988)$. In conclusion, it appears that inadequate cranialization of the tibia tuberosity might be an expected result of the TTA procedure, as well as persistent cranio-caudal instability during walking. However, instability cannot solely be attributed to insufficient cranialization because three out of nine unstable stifles were sufficiently or even overcorrected $\left(\mathrm{PTA} \leq 90^{\circ}\right)$. Further in vivo studies are needed to resolve these conflicting findings.
\end{abstract}

Keywords: Cruciate ligament rupture, Dog, Fluoroscopy, Joint stability, Kinematics.

\section{Introduction}

Ruptures of the cranial cruciate ligament are the most frequent reason for hind limb lameness in dogs (Lampman et al., 2003). Surgical methods available are extra-articular "cruciate ligament replacement techniques" (e.g. suture restraint mechanisms) and dynamic procedures (Knebel and Meyer-Lindenberg, 2014). The latter include tibial tuberosity advancement (TTA) (Kipfer et al., 2008). In this procedure the tibial crest is displaced cranially, with the aim of creating a patellar tendon angle (PTA) of $90^{\circ}$ to the tibial plateau (Montavon et al., 2002; Boudrieau, 2009). Ex vivo studies show that TTA with a PTA of $90^{\circ}$ reduces the cranio-caudal femoro-tibial subluxation in existing rupture of the cranial cruciate ligament (Apelt et al., 2007; Kipfer et al., 2008). While tibial plateau leveling osteotomy (TPLO) in vitro induces $6^{\circ}$ varus angulation and an increase in the medial femoro-tibial contact pressure (Kim et al., 2010), in TTA ex vivo the physiological femoro-tibial contact and pressure patterns are restored (Guerrero et al., 2011).

Based on subjective evaluation by surgeons, telephone interview of the owners and/or thigh circumference, dogs which were treated with TTA have been reported to show good to very good functional results (Lafaver et al., 2007; MacDonald et al., 2013). However, compared with TPLO, TTA tends to achieve a lower level of functional outcome (Krotscheck et al., 2016). In objective gait analyses determining the vertical ground reaction forces, it was shown that there was a significant improvement after TTA but that the original limb function was not restored completely (Voss et al., 2008; Skinner et al., 2013; Krotscheck et al., 2016). In spite of this, $84 \%$ of dog owners whose dog had been treated with TTA were satisfied and, if rupture of the cruciate ligament occurred again, would have TTA performed again (Steinberg et al., 2011).

Classical complications after TTA, in addition to infections and implant failure (e.g. plate or cage breakage, screws loosening) include tibial crest avulsion/fracture, tibial fissures/fractures as well as, above all, secondary meniscus damage (Lafaver $e t$ al., 2007; Stein and Schmoekel, 2008; Steinberg et al., 2011; Christopher et al., 2013). The incidence of postoperative secondary meniscus damage after TTA ranges between $8.5 \%$ and $21.7 \%$ (Lafaver et al., 2007; Stein and Schmoekel, 2008; Steinberg et al., 2011; Wolf et al., 2012). A possible reason for the relatively 
high number of secondary meniscal tears and for the failure of limb function to return to normal after TTA could be persistent cranio-caudal stifle instability (Skinner et al., 2013; Rey et al., 2014). Skinner et al. (2013) and Rey et al. (2014) found persistent postoperative subluxation and instability in vivo in 21 out of $30(70 \%)$ and 4 out of $6(67 \%)$ of cases of stifles treated with TTA. In doing so, Skinner et al. (2013) based their conclusions on medio-lateral $\mathrm{x}$-ray images of the stifle during standing. Rey et al. (2014) studied the stifles while the dogs were walking on a treadmill using uniplanar fluoroscopy and then subjective assessment of the high-speed video sequences obtained with regard to a visible cranio-caudal sliding motion between the femur and the tibia. In contrast to TPLO, in which Kim et al. (2012) found a connection between medial meniscectomy and postoperative subluxation during standing in a total of 15 animals, Skinner et al. (2013) were unable to confirm this effect in connection with TTA as $86 \%$ of the stifles had undergone partial meniscectomy at the time of the TTA operation.

In the study by Rey et al. (2014), which so far was the only one to investigate stifle stability during locomation, there is no corresponding data concerning postoperative meniscus status. There is also no information on what PTA the joints display after TTA. Therefore, it cannot be assessed whether any technical deficiencies when carrying out TTA (e.g. inadequate cranialization of the tibial tuberosity) are responsible for the high incidence of unstable joints after TTA in that specific report. In Skinner et al. (2013) the mean postoperative PTA was $89^{\circ}$, with a standard deviation of $3^{\circ}$. Consequently, approximately one third of the joints are considered to be undercorrected, which does not, however, explain the $70 \%$ incidence of unstable joints. Krotscheck et al. (2016) and Hoffmann et al. (2006) achieve a mean postoperative PTA of $91.9 \pm$ $2.7^{\circ}$ and $95.5^{\circ}\left(89.5\right.$ to $\left.101.5^{\circ}\right)$. Therefore it can be concluded that a not insignificant number of stifles do not achieve the target of $\mathrm{a} \leq 90^{\circ}$ PTA after TTA operation. Whether a PTA of $90^{\circ}$ is in fact sufficient to guarantee stability in vivo after TTA is debatable, as Hoffmann et al. (2011) required a PTA of $86^{\circ}$ ex vivo in the case of an increased load on the stifle in order to obtain a stable stifle situation. Furthermore, as has been demonstrated by Skinner et al. (2013), even stifles with adequate transposition may show femoro-tibial subluxation at the stance in vivo.

The aim of this study was to report the incidence of postoperative cranio-caudal stifle (in)stability following TTA during locomotion and to investigate the effect on stifle stability of postoperative PTA and, if applicable, of any meniscus surgery carried out. In contrast to Skinner et al. (2013) we assume that there are higher joint pressures in the moving rather than in the standing animal and, as a result, expect a higher number of unstable joints. In addition, we followed the hypothesis that the resection of unstable parts of the medial meniscus has no effect on postoperative stifle stability during locomotion.

\section{Animals}

\section{Material and Methods}

All dogs which were treated at the Small Animal Department of the University of Leipzig, Germany with a TTA between 2009 and 2012 for a complete rupture of the cranial cruciate ligament and which underwent gait analysis postoperatively by fluoroscopic kinematography were considered retrospectively. In addition, it was a prerequisite that a preoperative and postoperative $\mathrm{x}$-ray image of the joints was available for determining the PTA. It also had to be known whether there was meniscus damage and whether meniscal tissue had been resected during the operation.

\section{Clinical examination}

At the time of the TTA and of the fluoroscopic gait analysis, all patients were examined orthopedically by a surgical resident or diplomate. Written results of the orthopedic examination such as joint swelling and palpatory cranio-caudal stifle instability (drawer) were evaluated.

\section{Radiography and preoperative planning}

The preoperative planning was based on two orthogonal radiographs of the stifle in question in extension. In the medio-lateral radiographic projection, care was taken to achieve superimposition of the tibial and femoral condyles. The cage size needed was determined directly from the medio-lateral projection with the circle template "KTTA Pre-OP Planning Guide" (Kyon AG, Zürich, Switzerland) (Fig. 1), in which cage size was rounded up to the next largest size. The template is based on the common tangent method according to Dennler et al. (2006) and is described in more detail below: first of all the circle template was placed on the superimposed femoral condyles. Then the center of the circle was determined (Fig. 2).

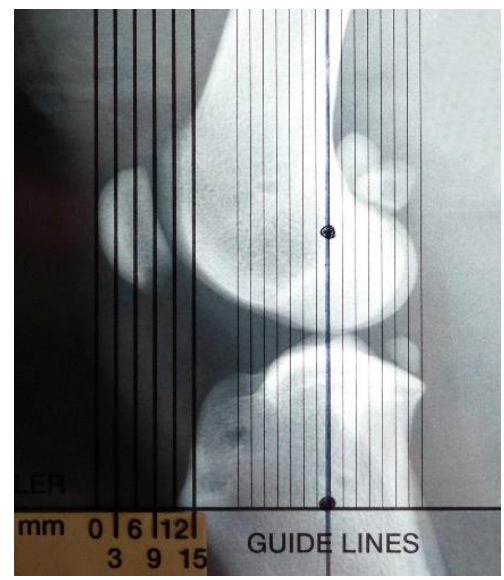

Fig. 1. Preoperative cage size planning using the 'KTTA PreOP Planning Guide' (Kyon AG, Zürich, Switzerland). 


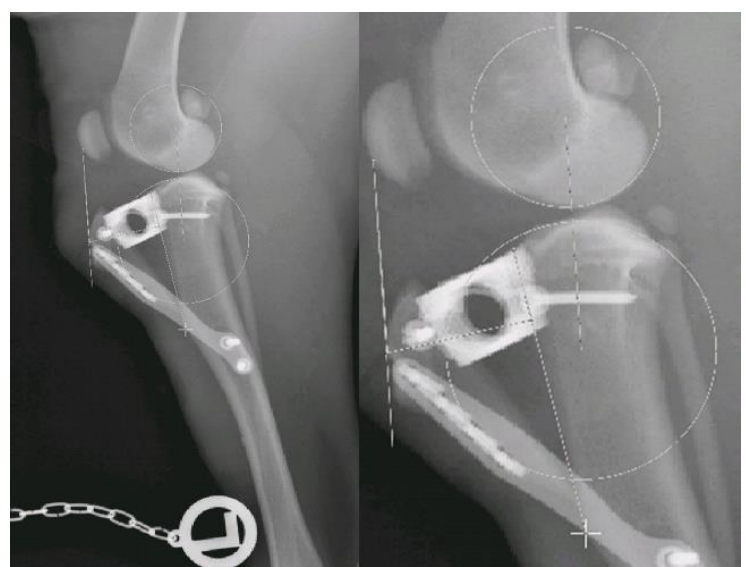

Fig. 2. Digital measurement of postoperative patellar tendon angle (PTA) using dedicated software within ParaView (www.paraview.org).

At this point it has to be emphasized that contrary to earlier measuring methods, we use the arc on the femoro-tibial contact surface for alignment with the template. This generally leads to the circle being larger and its center being positioned rather more cranially than when the circle template is placed on the contour of the caudal femoral condyle, as was the practice for a long time. This procedure corresponds to Kyon's current recommendation for the preoperative planning of TTA. In the case of incomplete superimposition of the condyles, identical but separate procedures were carried out for the medial and lateral condyles and the center points of their respective circles were determined. Half way along a connecting line between the two center points of the circles obtained in this way the "averaged" reference point was now established. After this, the circle template was laid on the medial tibial condyle, by matching the circle with the contour of the medial condylar surfaces. Finally, both midpoints, the femoral and the tibial one, were connected with a line and by applying the Kyon AG (Zürich, Switzerland) "KTTA Pre-OP Planning Guide", in accordance with the instructions for use, the respective cage size was determined. The idealized course of the patellar ligament ran from the cranial side of the tibial tuberosity to the cranial side of the patella. Anaesthesia and arthroscopy

According to the clinic's standard protocol, the animals were placed under general anesthesia. In addition, all the animals were given epidural anesthesia. For the operation the patient was in the supine position. After aseptic preparation of the operative field, each stifle was arthroscoped immediately before the TTA according to Böttcher et al. (2010a) and existing longitudinal and bucket-handle tears of the caudal horn of the medial meniscus were treated by resection of the unstable axial part. The Leipzig Stifle Distractor (KARL STORZ GmbH \& Co. KG, Tuttlingen, Germany) was used for optimal visualization of the medial meniscus (Böttcher et al., 2009; Winkels et al., 2016).

\section{Operative technique}

Following arthroscopy, medial access was gained to the proximal tibia, with mobilization of the sartorial muscle (caudal part) and of the aponeurosis of the gracilis, semimembranosus and semitendinosus muscles. After osteotomy of the tibial tuberosity, this was advanced cranially and the TTA cage of the pre-determined size (Kyon AG, Zürich, Switzerland) was inserted. Fixation of the cage and of the tibial crest was performed using commercially available TTA implants from Kyon AG (Zürich, Switzerland). The soft tissue was routinely closed in several layers. Refixation of the aponeurosis of the gracilis, semimembranosus und semitendinosus muscles was not carried out explicitly. Nor was any kind of plication of the joint capsule carried out. Only the arthroscopic portals medially and laterally to the patellar ligament were adapted with a knot suture. Postoperative aftercare consisted of six weeks of the dog strictly being kept on a lead and the administration of a NSAID for 10 to 14 days. The sutures where generally be removed after 10 days by the referring veterinary surgeon. None of the animals received physiotherapy or other special rehabilitation measures postoperatively.

\section{Fluoroscopic kinematography}

Uniplanar fluoroscopic kinematography was performed on the affected joint. The dogs walked on a canine treadmill (Model DC7, JOG A DOG, Ottawa Lake, Michigan, USA). The speed of the treadmill was set at 0.8 to $1.0 \mathrm{~m} / \mathrm{s}$ depending on the size of the dog and the degree of lameness. To allow the dogs to get used to the treadmill, the animals were given at least two minutes before fluoroscopy was started. In all cases, the beam was directed latero-laterally on the stifle, in order to enable accurate assessment of the cranio-caudal movement in the sagittal plane (Fig. 3).

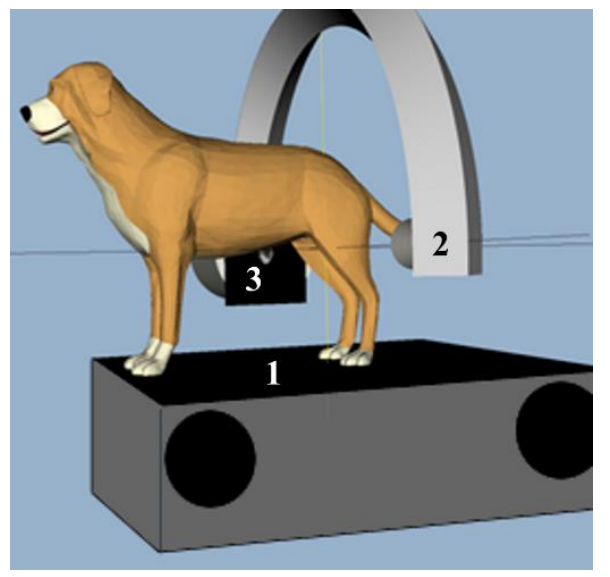

Fig. 3. Schematic of the experimental set-up during fluoroscopic kinematography. The dog is walking on the treadmill (1) while the x-ray tube (2) and the x-ray detector (3) are placed on each side of the animal. 
Depending on whether it was a left or right stifle, the direction of travel of the treadmill was set so that the index joint was on the side of the image detector. The fluoroscopy unit consisted of a standard C-arm ( BV 300, Philips Medical Systems Nederland B. V., Best, The Netherlands) with an in-built high-speed video camera (CamRecord 600, Optronis, Kehl, Germany), which replaced the standard video camera of the C-arm. During fluoroscopic kinematography the $\mathrm{C}$-arm worked in continuous mode at 80 to $110 \mathrm{kV}$ and 2.8 to $3.1 \mathrm{~mA}$ depending on the make-up of the body of the animal. The fluoroscopic sequences were recorded over 4 to 6 seconds at 500 images per second and an exposure time of $1 / 2000 \mathrm{~s}$. The sequences were selected according to whether the stifle affected had had at least three stance phases recorded, which were monitored by a second synchronized external high-speed video camera. The video sequences (Video 1 and 2) were checked visually for cranio-caudal translation between the femur and the tibia by 3 investigators independently. Finally, consensus was found in relation to stability (stable or unstable) and the pattern of movement (cranial - subluxation of the tibia vs. caudal slippage of the femur). The assessment of the pattern of movement is based on whether the distal femur visibly moved caudally or the proximal tibia visibly subluxated cranially. Every visually perceptible cranio-caudal movement between the femoral condyles and the tibial plateau was defined as an unstable stifle. Video sequences were available to train the investigators and were studied in the, among other things, healthy and cruciate ligament-insufficient stifles (Rey et al., 2014). As a result, it was possible to divide the video sequences available into the "stable" category, for those, which corresponded to the healthy cruciate ligament-intact stifles and into the "unstable" category for those videos, which corresponded to the cruciate ligament-insufficient stifles. The degree of instability was not quantified in this study. The stifles were also not examined for an already chronic, persistent femorotibial subluxation, in which no further cranio-caudal translation is taking place. Such stifles would be classified as stable in the context of the study.

\section{Patellar Tendon Angle (PTA)}

The retrospective determination of the preoperative and postoperative PTA was carried out on the basis of the digital x-ray projections performed under narcosis. To do this we used a self-programmed plugin (= modular software extension) in the Paraview program (paraview 4.1.0, www.kitware.com, USA). This made it easier to identify the landmarks for determining the common tangents between femoral condyles and the tibial plateau as well as the course of the patellar ligament (Fig. 2). The PTA was then calculated automatically by the plugin. A PTA of $>90^{\circ}$ has to be considered as undercorrection and a PTA of $<90^{\circ}$ as overcorrection.
The stifle angle was determined from the preoperative and postoperative medio-lateral radiographs. This was measured as the angle at the intersection of two straight lines drawn axially through the respective medullary cavities of the femur and the tibia.

\section{Statistics}

The data was analyzed in MedCalc (MedCalc Software, Belgium). The normality of the continuous data was confirmed by the D'Agostino-Pearson test. The values are presented accordingly as the mean with standard deviation plus the minimum and maximum value. The influence of meniscus surgery, cage size and postoperative PTA on the stability achieved was determined with a logistic regression. For all tests, the significance level was $\leq 0.05$.

\section{Results}

\section{Epidemiological data}

In the period in question twelve dogs with complete rupture of the cranial cruciate ligament were treated with TTA at the Department of Small Animal Medicine of the Leipzig University, Germany. Of these, eight dogs with a total of ten operated stifles (6 unilateral, 2 bilateral) had a follow-up examination in the clinic and were examined accordingly by fluoroscopy (Table 1). All ten stifles met the criteria required to be included in the current study, with the data on two stifles coming from the study by Rey et al. (2014). The mean age of the eight animals examined was $4.7 \pm 3.0$ years (MinMax: 1.1 - 9.5). Their mean weight was $27.3 \pm 8.9 \mathrm{~kg}$ (Min-Max: 15.7 - 40.0). There were two male and six female dogs. The majority were large breeds dogs in which crossbreeds $(n=3)$ and Retriever breeds $(n=2)$ dominated. The remaining three dogs were a Beagle, a Bull Terrier and a Russian Terrier.

\section{Clinical findings}

Preoperatively all animals had a positive drawer test, increased stifle effusion and clear lameness. The clinical records available did not contain any more precise details on the degree of lameness at the time of operation, which would have allowed reliable determination of lameness score pre- and postoperatively in this retrospective study.

\section{Operative findings}

In all patients arthroscopy confirmed complete rupture of the cranial cruciate ligament. Three stifles displayed a medial bucket-handle tear (Table 1), in which in one stifle there was also a bucket-handle tear of the lateral meniscus. There was an isolated radial tear of the lateral meniscus in a further stifle, which was not treated further surgically. The remaining six stifles did not show any meniscal damage. There was no meniscal release in any of the ten stifles performed. Cages of 10.5 $\mathrm{mm}(\mathrm{n}=3)$ and $15.0 \mathrm{~mm}(\mathrm{n}=3)$ were the ones most frequently inserted. In addition, the $7.5 \mathrm{~mm}$ and $9.0 \mathrm{~mm}$ cages were each used in two stifles (Table 1). No intraoperative or postoperative complications recorded. 


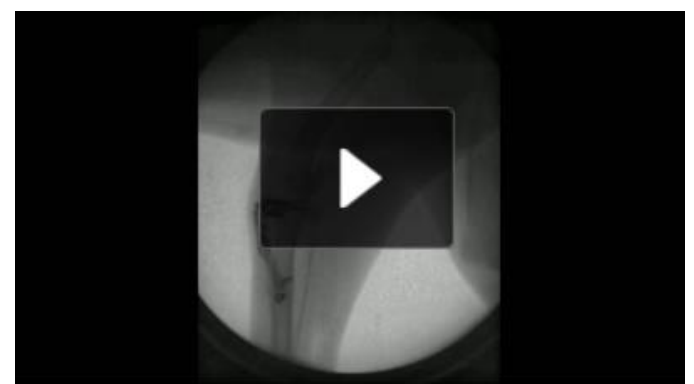

Video 1. Sagittal kinematographic sequence of a 3-year-old female crossbreed dog with complete rupture of the cranial cruciate ligament and TTA (patient $4, \mathrm{PTA}=98.5^{\circ}$ ). During stance phase, no cranio-caudal translation between femur and tibia is visible. Therefore, this stifle has to be classified as 'stable'. Note: This animal underwent implantation of tantalum beads for a separate study investigating threedimensional stifle kinematics (Reg. No.: TVV 07/14, Landesdirektion Sachsen). (https://www.openveterinaryjournal.com/videos).

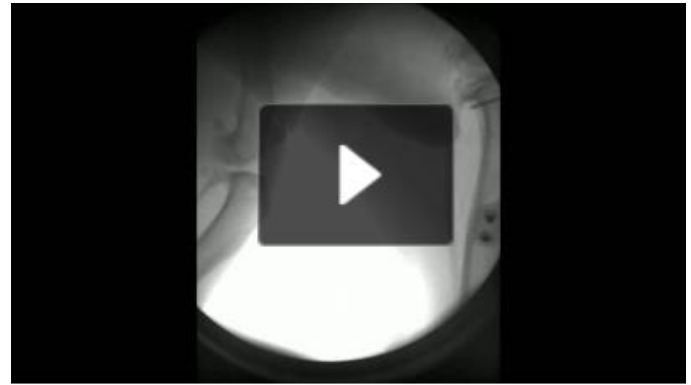

Video 2. Sagittal fluoroscopic kinematography of a 7-yearold male crossbreed dog after TTA (patient 7a, PTA $=86.6^{\circ}$ ). Note the caudal translation of the femoral condyles during early stance phase. Therefore, persistent instability is present in the stifle. (https://www.openveterinaryjournal.com/videos).

\section{Fluoroscopic kinematography}

The postoperative gait analysis took place on average $12.8 \pm 8.4$ weeks (Min-Max: $5.4-28.4$ ) after TTA. In this, nine of the ten stifles examined displayed visible cranio-caudal translation and are therefore to be assessed as unstable (Tables 1 and 2; Video 1 and 2). Therefore, there was persisting instability after TTA in $90 \%$ of the stifles examined.

\section{Postoperative Patellar Tendon Angle (PTA)}

The mean extension angle on the preoperative planning images was $129.3 \pm 12.4^{\circ}$ (Min-Max: $108.0^{\circ}-144.0^{\circ}$ ) and on the postoperative X-ray images it was $129.4 \pm$ $8.8^{\circ}$ (Min-Max: $112.0^{\circ}-146.0^{\circ}$ ).

The mean PTA after TTA was $92.4 \pm 3.6^{\circ}$ (Min-Max: $86.6^{\circ}-98.5^{\circ}$ ). Three stifles were considered to be overcorrected $\left(88.4 \pm 1.6^{\circ}\right.$; Min-Max: $\left.86.6^{\circ}-89.6^{\circ}\right)$. In contrast, the remaining seven stifles were considered to be undercorrected with a PTA of $>90^{\circ}\left(94.2 \pm 2.5^{\circ}\right.$; Min-Max: $91.2^{\circ}-98.5^{\circ}$ ).

The only joint that was stable in the fluoroscopic analysis had a PTA of $98.5^{\circ}$ and is therefore clearly undercorrected (Table 1).

\section{Logistic regression analysis}

None of the three variables examined (meniscus resection, postoperative PTA, cage size) showed a significant influence on postoperative stifle stability in vivo $(\mathrm{p}=0.0988)$.

\section{Discussion}

The dynamic stabilization of a cruciate ligamentinsufficient stifle with TTA is based on the concept that the tibio-femoral shearing forces are neutralized by changing the vector of the patellar ligament force by cranialization of the tibial tuberosity (Boudrieau, 2009). In vitro the TTA technique successfully restores cranio-caudal stability at a PTA of $\leq 90^{\circ}$ (Apelt et al., 2007). The results of this study do, however, show that in movement (in vivo), it is not necessary for stability to be present after TTA. The slightly higher number of unstable stifles (90\%) compared to the study of Skinner et al. (2013) (70 \%) supports our hypothesis that possible compensatory mechanisms, which are able to have a stabilizing effect during stance, fail in the presence of the forces occurring under locomotion. Even if the functional result after TTA in the current study was out of scope and the clinical records lack detailed data on the degree of lameness before and after TTA, there was the subjective impression that lameness improved in spite of persistent instability. This observation is in accordance with the force plate data reported by others (Voss et al., 2008; Skinner et al., 2013; Krotscheck et al., 2016). Therefore, based on the herewith reported accumulating evidence of persistent stifle instability after TTA, we propose to differentiate between mechanical efficacy and clinical outcome following TTA in the future, as both might not be inherently interconnected, which contradicts our current understanding of stifle biomechanics so far. Such conflicting findings are not unique to TTA, as Kim et al. (2012) report stifle joint instability following TPLO, too, but the number of instable stifles seems to be higher with TTA than with TPLO. Because neither the tibial compression tests nor cranial tibial thrust are suitable tests to intraoperatively evaluate a stifle with TTA for its expected stability in vivo, surgeons might falsely equate their subjective clinical impression of functional improvement after TTA with mechanical stability of the stifle. Contrary to our expectations, the target of a PTA of $\leq 90^{\circ}$ could not be met in the majority of cases we operated, which immediately suggests that the high number of unstable joints after TTA is connected with the high number of joints considered undercorrected ( 7 out of 10 joints). This is probably particularly due to the difficulty of determining the necessary cage size correctly (Cadmus et al., 2014), in which, in general, too small cage sizes are calculated in the preoperative planning (Bush et al., 2011; Etchepareborde et al., 2011; Millet et al., 2013; Bismuth et al., 2014; Cadmus et al., 2014). 
Table 1. Clinical data, signalment, meniscal findings, cage size, preoperative and postoperative stifle angle and patellar tendon angle (PTA), in vivo stability and period between surgery and kinematography ( 8 dogs, 10 stifles).

\begin{tabular}{|c|c|c|c|c|c|c|c|c|c|c|c|c|c|}
\hline \multirow{2}{*}{ Case } & \multirow{2}{*}{ Breed } & \multirow{2}{*}{$\begin{array}{c}\text { Age } \\
\text { (years) }\end{array}$} & \multirow{2}{*}{ Sex } & \multirow{2}{*}{$\begin{array}{l}\text { Weight } \\
(\mathrm{kg})\end{array}$} & \multirow{2}{*}{ Side } & \multirow{2}{*}{ Menisci } & \multirow{2}{*}{ Cage } & \multicolumn{2}{|c|}{ Stifle angle ${ }^{\circ}$} & \multicolumn{2}{|c|}{$\mathrm{PTA}^{\circ}$} & \multirow{2}{*}{ Stability } & \multirow{2}{*}{ Time* } \\
\hline & & & & & & & & Pre-OP & Post-OP & Pre-OP & Post-OP & & \\
\hline 1a & $\begin{array}{l}\text { Labrador } \\
\text { Retriever }\end{array}$ & 2.5 & $\mathrm{~F}$ & 30.0 & right & $\begin{array}{l}\text { Radial tear of } \\
\text { the lateral } \\
\text { caudal horn }\end{array}$ & 10.5 & 120 & 130 & 99.2 & 91.2 & Unstable & 8.7 \\
\hline $1 b$ & $\begin{array}{l}\text { Labrador } \\
\text { Retriever }\end{array}$ & 2.5 & $\mathrm{~F}$ & 30.0 & left & no findings & 10.5 & 126 & 130 & 100.6 & 95.8 & Unstable & 12.4 \\
\hline 2 & $\begin{array}{c}\text { Russian } \\
\text { Terrier }\end{array}$ & 1.7 & $\mathrm{~F}$ & 40.0 & right & no findings & 9.0 & 129 & 132 & 100.8 & 92.2 & Unstable & 28.4 \\
\hline 3 & $\begin{array}{l}\text { Golden } \\
\text { Retriever }\end{array}$ & 3.9 & $\mathrm{~F}$ & 35.0 & left & no findings & 10.5 & 108 & 131 & 98.9 & 89.6 & Unstable & 12.3 \\
\hline 4 & Crossbreed & 3.2 & $\mathrm{~F}$ & 15.7 & right & no findings & 7.5 & 144 & 130 & 101.2 & 98.5 & Stable & 5.4 \\
\hline 5 & Beagle & 7.8 & M & 18.6 & right & no findings & 9.0 & 140 & 121 & 103.8 & 94.7 & Unstable & 19.0 \\
\hline 6 & Crossbreed & 9.5 & $\mathrm{~F}$ & 16.0 & right & no findings & 7.5 & 139 & 127 & 100.3 & 92.1 & Unstable & 22.0 \\
\hline $7 \mathrm{a}$ & Crossbreed & 7.1 & M & 34.0 & right & $\begin{array}{l}\text { Bucket- } \\
\text { handle tear } \\
\text { medial }\end{array}$ & 15.0 & 113 & 112 & 100.6 & 86.6 & Unstable & 6.1 \\
\hline $7 b$ & Crossbreed & 7.3 & M & 34.0 & left & $\begin{array}{l}\text { Bucket- } \\
\text { handle tear } \\
\text { medial }\end{array}$ & 15.0 & 133 & 135 & 103.8 & 94.6 & Unstable & 7.3 \\
\hline 8 & $\begin{array}{l}\text { Bull } \\
\text { Terrier }\end{array}$ & 1.1 & $\mathrm{~W}$ & 20.0 & links & $\begin{array}{l}\text { Bucket- } \\
\text { handle tear } \\
\text { medial and } \\
\text { lateral }\end{array}$ & 15.0 & 141 & 146 & 102.5 & 89.0 & Unstable & 6.0 \\
\hline
\end{tabular}

* Time between TTA and fluoroscopic kinematography.

Table 2. Crosstabulation of the patellar tendon angle (PTA) in view of the cranio-caudal stability of the stifle after TTA.

\begin{tabular}{cccc}
\hline PTA & \multicolumn{2}{c}{ Fluoroscopic gait analysis } & Frequency \\
\cline { 2 - 3 } & stable & unstable & $(\%)$ \\
\hline$>90^{\circ}$ & 1 & 6 & 70.0 \\
$90^{\circ}$ & 0 & 0 & 0 \\
$<90^{\circ}$ & 0 & 3 & 30.0 \\
\hline Frequency $(\%)$ & 10.0 & 90.0 & \\
\hline
\end{tabular}

Known influencing factors in this are the extension angle of the joint and a non-exact medio-lateral radiographic projection, which can cause varying measurement results (Bush et al., 2011; Hoffmann et al., 2011; Millet et al., 2013; Bismuth et al., 2014). In our population we have to state that the extension angle of the preoperative radiographs for a reliable determination of the cage size was, on average, smaller than the measurement required of $135^{\circ}$ (Damur et al., 2003; Dennler et al., 2006; Bush et al., 2011). This results in the selection of cage sizes which are too small and therefore in potential undercorrection.

Despite the technical limitations in performing the TTA technique in our patient population and the small number of stifles investigated, we believe that our results are to be regarded as being representative for other dogs with TTA for the following reasons. Other authors too refer to a patient population with potentially undercorrected stifles. After TTA in 65 dogs Hoffmann et al. (2011) found a mean postoperative PTA of $95.5^{\circ}$. Krotscheck et al. (2016) and Skinner et al. (2013) also report on a large number of potentially undercorrected stifles (PTA $91.9 \pm 2.7^{\circ}$ and $89 \pm 3.0^{\circ}$ respectively). Another reason is the unlikelihood of selection bias, which otherwise might have resulted in a patient population containing mostly particularly "poor" TTA cases. Neither the subjectively observed overall improvement in limb function following TTA, which parallels the experiences reported by others (Lafaver $e t$ al., 2007; MacDonald et al., 2013), nor the absence of surgical complications in our study population, would support such conclusion. Similar applies to the relatively high incidence of persistent postoperative stifle instability in the current study. Somehow it was apprehended to observe a high percentage of postoperative instability, because Skinner et al. (2013), Rey et al. (2014) as well as the ex vivo tests by Hoffmann et al. (2011) already showed that in spite of TTA a certain proportion of operated stifles continue to be unstable in movement.

In summary, we notice that the mechanical concept of TTA, which is based on dynamic stabilization of the stifle joint during weight-bearing appears to achieve joint stability less reliably in-vivo than what is commonly postulated, based on overall clinical function and/or cadaveric testing. 
The decision to record the kinematographic data relatively early after the operation $(12.8 \pm 8.4$ weeks $)$ is in direct relation to the observation by Tashman et al. (2004), who showed that after iatrogenic cranial cruciate ligament rupture there is a reduction in the amount of cranio-caudal instability with time. This is due to increasing, chronic femoro-tibial subluxation. Therefore the extent of the femoro-tibial translation reduces with time and it was to be feared that, with a longer monitoring period, the assessment of the craniocaudal sliding motion would either not be seen or would only be seen with difficulty in the fluoroscopic highspeed video sequences. Even if with the present study we cannot rule out the possibility that the rate of stable, but progressively subluxated, stifles would have increased with a longer monitoring period, the results of Skinner et al. (2013) put this hypothesis into perspective as they examined the patients after $18 \pm 14$ months and obtained similar results.

It was noticeable among our animals that the only dog with a stable stifle, compared with the other test subjects, had a relatively low body weight $(16 \mathrm{~kg})$. One possible explanation for this would be that the muscle strength and lever arms in vitro, unlike in the in vivo situation, differ in movement in medium-sized and large dogs. Indeed, Hoffmann et al. (2011) noted that a PTA of $<86^{\circ}$ (clear overcorrection) has to be achieved to attain cranio-caudal stability under increased stifle load (around 50\%). Whether there is a difference between small and medium-sized or large dogs in the treatment with TTA is unknown so far.

Beside the overall incidence of postoperative stifle instability and the impact of post-operative PTA, we were interested in the effect of meniscal surgery on joint stability following TTA. Four out of ten stifles investigated displayed medial meniscus lesions. All four stifles displayed postoperative instability. However, instability was also found in the majority of stifles which had intact menisci. Therefore, it appears that, at least during movement, the menisci have no significant role in stifle stability after TTA. This contrasts with the observation by Kim et al. (2012), which after TPLO observed an in vivo stabilizing effect of the menisci in the standing animal.

This study shows methodological limitations that should be borne in mind when interpreting the results. In particular, the low number of cases and the small number of stable stifles postoperatively made it difficult to carry out a profound data analysis. It is possible that significant correlations could not be demonstrated because of a type 2 error combined with the low power of the study due to the one-sided data situation. Furthermore, the binary code for the assessment of stifle stability (stable vs. unstable) may be an unnatural assessment of joint mechanics. As described by Rey et al. (2014), no translation can be detected visually in kinematographic video sequences in healthy dogs. This can also be seen by inexperienced investigators from film sequences 1 and 2 . However, this does not rule out the fact that small translations could be perceived as being stable (false negative assessment).

On the other hand, there may be a degree of femorotibial translation, which can be seen visually under fluoroscopy, but because of the relatively small extent (of the order of $3-5 \mathrm{~mm}$ ) might not comparable with a "total" femoro-tibial subluxation of 8-15 mm, as occurs after severing the cranial cruciate ligament without stabilization (Tashman and Anderst, 2003; Böttcher et al., 2010b). It can be assumed that the extent of the translation movement is significant for the stifle function and secondary damage, such as meniscal tears, after cruciate ligament rupture (Wegrzyn et al., 2009). The technique established by Kim et al. (2011) to measure femoro-tibial translation in millimeters could not be used in this study as the quality of the fluoroscopic video sequences made precise determination of the landmarks, in particular in the area of the tibial plateau, impossible. For future studies, it would be desirable to develop a similar method, which would make the quantification of cranio-caudal subluxation possible from fluoroscopic images.

The investigation on the treadmill itself can be assessed as a further possible influencing factor. Walking on a treadmill can lead to changes in gait pattern (Fredricson et al., 1983; Buchner et al., 1994). In people, therefore, a period of 6 to 8 minutes is selected for acclimatization until there is a normal gait pattern apparent (Schieb, 1986; Matsas et al., 2000), whereas in dogs two minutes are considered to be sufficient (Vilensky et al., 1994a, 1994b; Owen et al., 2004; Fanchon et al., 2006). Therefore, before the images were recorded, all the animals were allowed to acclimatize for at least two minutes, but we cannot exclude completely some alterations in gait, which might have biased our findings when compared to natural over ground motion This study shows a high number of potentially undercorrected stifles, which we attribute to inadequate preoperative planning and the associated choice of cages size being too small in the majority of the stifles operated.

On the other hand, both over- and undercorrected stifles remained unstable postoperatively and it appears to be generally debatable whether cranial transposition of the tibial tuberosity in vivo is able to predictably stabilize cranial cruciate ligament-insufficient stifles. A final judgment on this can, therefore, only be made if a larger number of cranial cruciate ligament-insufficient stifles with adequate cranialization of the tibial tuberosity are evaluated.

\section{Conflict of interest}

The Authors declare that there is no conflict of interest. 


\section{References}

Apelt, D., Kowaleski, M.P. and Boudrieau, R.J. 2007. Effect of Tibial Tuberosity Advancement on Cranial Tibial Subluxation in Canine Cranial Cruciate-Deficient Stifle Joints: An In Vitro Experimental Study. Vet. Surg. 36, 170-177. doi.org/10.1111/j.1532-950X.2007.00250.x.

Bismuth, C., Ferrand, F.X., Millet, M., Labrunie, A., Marin, B., Pillard, P., Deroy, C., Fau, D., Carozzo, C., Cachon, T. and Viguier, E. 2014. Comparison of radiographic measurements of the patellar tendontibial plateau angle with anatomical measurements in dogs. Validity of the common tangent and tibial plateau methods. Vet. Comp. Orthop. Traumatol. 27, 222-229.

Böttcher, P., Winkels, P. and Oechtering, G. 2009. A novel pin distraction device for arthroscopic assessment of the medial meniscus in dogs. Vet. Surg. 38, 595-600.

Böttcher, P., Brühschwein, A., Winkels, P., Werner, H., Ludewig, E., Grevel, V. and Oechtering, G. 2010a. Value of low-field MRI in diagnosing meniscal tears in the canine stifle: a prospective study evaluating sensitivity and specificity in naturally occurring cranial cruciate ligament deficiency with arthroscopy as the gold standard. Vet. Surg. 39, 296-305.

Böttcher, P., Fischer, C., Werner, H., Grevel, V. and Oechtering, G. 2010b. Stifle stability after lateral suture stabilisation using Ethibond $\operatorname{Excel}(\mathrm{R})$ : early destabilisation following cyclic passive joint motion. Tierarztl Prax Ausg K Kleintiere Heimtiere 38(2), 61-69.

Boudrieau, R.J. 2009. Tibial Plateau Leveling Osteotomy or Tibial Tuberosity Advancement? Vet. Surg. 38, 1-22.

Buchner, H.H., Savelberg, H.H., Schamhardt, H.C., Merkens, H.W. and Barneveld, A. 1994. Kinematics of treadmill versus overground locomotion in horses. Vet. Q. 16(Suppl. 2), S87-90.

Bush, M.A., Bowlt, K., Gines, J.A. and Owen, M.R. 2011. Effect of use of different landmark methods on determining stifle angle and on calculated tibial tuberosity advancement. Vet. Comp. Orthop. Traumatol. 24, 205-210.

Cadmus, J., Palmer, R.H. and Duncan, C. 2014. The effect of preoperative planning method on recommended tibial tuberosity advancement cage size. Vet. Surg. 43, 995-1000.

Christopher, S.A., Beetem, J. and Cook, J.L. 2013. Comparison of long-term outcomes associated with three surgical techniques for treatment of cranial cruciate ligament disease in dogs. Vet. Surg. 42, 329-334.

Damur, D., Tepic, S. and Montavon, P. 2003. Proximal tibial osteotomy for the repair of cranial cruciate- deficient stifle joints in dogs. Vet. Comp. Orthop. Traum. 16, 211-216.

Dennler, R., Kipfer, N.M., Tepic, S., Hassig, M. and Montavon, P.M. 2006. Inclination of the patellar ligament in relation to flexion angle in stifle joints of dogs without degenerative joint disease. Am. J. Vet. Res. 67, 1849-1854.

Etchepareborde, S., Mills, J., Busoni, V., Brunel, L. and Balligand, M. 2011. Theoretical discrepancy between cage size and efficient tibial tuberosity advancement in dogs treated for cranial cruciate ligament rupture. Vet. Comp. Orthop. Traumatol. 24, 27-31.

Fanchon, L., Valette, J.P., Sanaa, M. and Grandjean, D. 2006. The measurement of ground reaction force in dogs trotting on a treadmill: an investigation of habituation. Vet. Comp. Orthop. Traumatol. 19, 8186.

Fredricson, I., Drevemo, S., Dalin, G., HjertéN, G., BjöRne, K., Rynde, R. and Franzen, G. 1983. Treadmill for equine locomotion analysis. Equine Vet. J. 15, 111-115.

Guerrero, T.G., Pozzi, A., Dunbar, N., Kipfer, N., Haessig, M., Beth Horodyski, M. and Montavon, P.M. 2011. Effect of Tibial Tuberosity Advancement on the Contact Mechanics and the Alignment of the Patellofemoral and Femorotibial Joints. Vet. Surg. 40, 839-848.

Hoffmann, D.E., Miller, J.M., Ober, C.P., Lanz, O.I., Martin, R.A. and Shires, P.K. 2006. Tibial tuberosity advancement in 65 canine stifles. Vet. Comp. Orthop. Traumatol. 19, 219-227.

Hoffmann, D.E., Kowaleski, M.P., Johnson, K.A., Evans, R.B. and Boudrieau, R.J. 2011. Ex vivo biomechanical evaluation of the canine cranial cruciate ligament-deficient stifle with varying angles of stifle joint flexion and axial loads after tibial tuberosity advancement. Vet. Surg. 40, 311320.

Kim, S.E., Pozzi, A., Banks, S.A., Conrad, B.P. and Lewis, D.D. 2010. Effect of cranial cruciate ligament deficiency, tibial plateau leveling osteotomy, and tibial tuberosity advancement on contact mechanics and alignment of the stifle in flexion. Vet. Surg. 39, 363-370.

Kim, S.E., Lewis, D.D., Pozzi, A., Seibert, R.L. and Winter, M.D. 2011. Radiographic quantitative assessment of cranial tibial subluxation before and after tibial plateau leveling osteotomy in dogs. Am. J. Vet. Res. 72, 410-416.

Kim, S.E., Lewis, D.D. and Pozzi, A. 2012. Effect of tibial plateau leveling osteotomy on femorotibial subluxation: in vivo analysis during standing. Vet. Surg. 41, 465-470.

Kipfer, N.M., Tepic, S., Damur, D.M., Guerrero, T., Hassig, M. and Montavon, P.M. 2008. Effect of 
tibial tuberosity advancement on femorotibial shear in cranial cruciate-deficient stifles. An in vitro study. Vet. Comp. Orthop. Traumatol. 21(5), 385390.

Knebel, J. and Meyer-Lindenberg, A. 2014. Aetiology, pathogenesis, diagnosis and therapy of cranial cruciate ligament rupture in dogs. Tierarztl Prax Ausg K Kleintiere Heimtiere 42(1), 36-47.

Krotscheck, U., Nelson, S.A., Todhunter, R.J., Stone, M. and Zhang, Z. 2016. Long Term Functional Outcome of Tibial Tuberosity Advancement vs. Tibial Plateau Leveling Osteotomy and Extracapsular Repair in a Heterogeneous Population of Dogs. Vet. Surg. 45, 261-268.

Lafaver, S., Miller, N.A., Stubbs, W.P., Taylor, R.A. and Boudrieau, R.J. 2007. Tibial Tuberosity Advancement for Stabilization of the Canine Cranial Cruciate Ligament-Deficient Stifle Joint: Surgical Technique, Early Results, and Complications in 101 Dogs. Vet. Surg. 36, 573-586.

Lampman, T.J., Lund, E.M. and Lipowitz, A.J. 2003. Cranial Cruciate disease: current status of diagnosis, surgery and risk of disease. Vet. Comp. Orthop. Traumatol. 16(3), 122-126.

MacDonald, T.L., Allen, D.A. and Monteith, G.J. 2013. Clinical assessment following tibial tuberosity advancement in 28 stifles at 6 months and 1 year after surgery. Can. Vet. J. 54(3), 249-254.

Matsas, A., Taylor, N. and McBurney, H. 2000. Knee joint kinematics from familiarised treadmill walking can be generalised to overground walking in young unimpaired subjects. Gait Posture 11(1), 46-53.

Millet, M., Bismuth, C., Labrunie, A., Marin, B., Filleur, A., Pillard, P., Sonet, J., Cachon, T. and Etchepareborde, S. 2013. Measurement of the patellar tendon-tibial plateau angle and tuberosity advancement in dogs with cranial cruciate ligament rupture. Reliability of the common tangent and tibial plateau methods of measurement. Vet. Comp. Orthop. Traumatol. 26, 469-478.

Montavon, P.M., Damur, D.M. and Tepic, S. 2002. Advancement of the tibial Tuberosity for the treatment of cranial cruciate deficient canine stifle. In: Proceedings of $1^{\text {st }}$ World Orthopedic Veterinary Congress. Munich, Germany, pp: 152.

Owen, M.R., Richards, J., Clements, D.N., Drew, S.T., Bennett, D. and Carmichael, S. 2004. Kinematics of the elbow and stifle joints in greyhounds during treadmill trotting - An investigation of familiarisation. Vet. Comp. Orthop. Traum. 17, 141-145.

Rey, J., Fischer, M.S. and Bottcher, P. 2014. Sagittal joint instability in the cranial cruciate ligament insufficient canine stifle. Caudal slippage of the femur and not cranial tibial subluxation. Tierarztl Prax Ausg K Kleintiere Heimtiere 42(3), 151-156.

Schieb, D.A. 1986. Kinematic Accommodation of Novice Treadmill Runners. Res. Quart. Exerc. Sport 57, 1-7.

Skinner, O.T., Kim, S.E., Lewis, D.D. and Pozzi, A. 2013. In vivo femorotibial subluxation during weight-bearing and clinical outcome following tibial tuberosity advancement for cranial cruciate ligament insufficiency in dogs. Vet. J. 196, 86-91.

Stein, S. and Schmoekel, H. 2008. Short-term and eight to 12 months results of a tibial tuberosity advancement as treatment of canine cranial cruciate ligament damage. J. Small Anim. Pract. 49, 398404.

Steinberg, E.J., Prata, R.G., Palazzini, K. and Brown, D.C. 2011. Tibial tuberosity advancement for treatment of CrCL injury: complications and owner satisfaction. J. Am. Anim. Hosp. Assoc. 47, 250257.

Tashman, S. and Anderst, W. 2003. In-vivo measurement of dynamic joint motion using high speed biplane radiography and CT: application to canine ACL deficiency. J. Biomech. Eng. 125, 238245.

Tashman, S., Anderst, W., Kolowich, P., Havstad, S. and Arnoczky, S. 2004. Kinematics of the ACLdeficient canine knee during gait: serial changes over two years. J. Orthop. Res. 22, 931-941. doi.org/10.1016/j.orthres.2004.01.008.

Vilensky, J.A., O'Connor, B.L., Brandt, K.D., Dunn, E.A. and Rogers, P.I. 1994a. Serial kinematic analysis of the trunk and limb joints after anterior cruciate ligament transection: Temporal, spatial, and angular changes in a canine model of osteoarthritis. J. Electromyogr. Kinesiol. 4, 181192.

Vilensky, J.A., O'Connor, B.L., Brandt, K.D., Dunn, E.A., Rogers, P.I. and DeLong, C.A. 1994b. Serial kinematic analysis of the unstable knee after transection of the anterior cruciate ligament: temporal and angular changes in a canine model of osteoarthritis. J. Orthop. Res. 12, 229-237.

Voss, K., Damur, D.M., Guerrero, T., Haessig, M. and Montavon, P.M. 2008. Force plate gait analysis to assess limb function after tibial tuberosity advancement in dogs with cranial cruciate ligament disease. Vet. Comp. Orthop. Traumatol. 21, 243249.

Wegrzyn, J., Chouteau, J., Philippot, R., Fessy, M.H. and Moyen, B. 2009. Repeat revision of anterior cruciate ligament reconstruction: a retrospective review of management and outcome of 10 patients with an average 3-year follow-up. Am. J. Sports Med. 37, 776-785. 
Winkels, P., Pozzi, A., Cook, R. and Bottcher, P. 2016. Prospective Evaluation of the Leipzig Stifle Distractor. Vet. Surg. 45, 631-635.

Wolf, R.E., Scavelli, T.D., Hoelzler, M.G., Fulcher, R.P. and Bastian, R.P. 2012. Surgical and postoperative complications associated with tibial tuberosity advancement for cranial cruciate ligament rupture in dogs: 458 cases (2007-2009). J. Am. Vet. Med. Assoc. 240(12), 1481-1487. doi.org/10.2460/javma.240.12.1481. 